\title{
Use and Adoption of an Assisted Cognition System to Support Therapies for People with Dementia
}

\author{
René F. Navarro, ${ }^{1}$ Marcela D. Rodríguez, ${ }^{2}$ and Jesús Favela ${ }^{3}$ \\ ${ }^{1}$ Industrial Engineering Department, Universidad de Sonora, 83200 Hermosillo, SON, Mexico \\ ${ }^{2}$ School of Engineering, UABC, 21100 Mexicali, BC, Mexico \\ ${ }^{3}$ Department of Computer Science, CICESE, 22860 Ensenada, BC, Mexico
}

Correspondence should be addressed to René F. Navarro; rnavarro@industrial.uson.mx

Received 20 February 2016; Revised 11 July 2016; Accepted 1 August 2016

Academic Editor: Pietro Cipresso

Copyright (c) 2016 René F. Navarro et al. This is an open access article distributed under the Creative Commons Attribution License, which permits unrestricted use, distribution, and reproduction in any medium, provided the original work is properly cited.

\begin{abstract}
The cognitive deficits in persons with dementia $(\mathrm{PwD})$ can produce significant functional impairment from early stages. Although memory decline is most prominent, impairments in attention, orientation, language, reasoning, and executive functioning are also common. Dementia is also characterized by changes in personality and behavioral functioning that can be very challenging for caregivers and patients. This paper presents results on the use and adoption of an assisted cognition system to support occupational therapy to address psychological and behavioral symptoms of dementia. During 16 weeks, we conducted an in situ evaluation with two caregiver-PwD dyads to assess the adoption and effectiveness of the system to ameliorate challenging behaviors and reducing caregiver burden. Evaluation results indicate that intervention personalization and a touch-based interface encouraged the adoption of the system, helping reduce challenging behaviors in PwD and caregiver burden.
\end{abstract}

\section{Introduction}

As people age, there are increased risks of functional decay or developing a disease. For example, after the age of 65 , the chance of developing some form of dementia doubles approximately every five years. By the year 2010, there were 35.6 million people with dementia worldwide. It is predicted that by 2030 this number will increase to 65.7 million and to 115.4 million by 2050 [1]. Dementia is characterized by the loss of intellectual functions to the extent that it interferes with daily activities [2]. For instance, memory difficulties in $\mathrm{PwD}$ can impact self-confidence, leading to withdrawal from day-to-day activities, anxiety, and depression. Family caregivers are also affected due to the practical impact of memory problems on everyday life and to the strain of frustration that can result from it [3]. Besides cognitive decline, PwD presents several noncognitive symptoms, also known as challenging behaviors [4], which are problematic not only for the person with dementia but also for their caregivers. Behavioral and psychological symptoms of dementia (BPSD) are defined as "symptoms of disturbed perception, thought content, mood, behavior frequently occurring in patients with dementia" [5]. Psychological symptoms of dementia relate to anxiety, depression, and psychosis whereas behavioral symptoms include aggression, apathy, agitation, disinhibited behaviors, wandering, nocturnal disruption, and vocally disruptive behaviors. Such behaviors are typically identified by observation of the $\mathrm{PwD}$ and only considered challenging when they affect other people or cause self-injury. For instance, apathy commonly perceived as a lack of interest, emotion, and motivation is a neuropsychiatric syndrome whose prevalence reaches $93 \%$ of people with Alzheimer's disease [6]. These challenging behaviors are associated with high levels of distress in both $\mathrm{PwD}$ and their caregivers.

The care for a PwD is a complex and challenging task since the evolution of dementia involves progressive decline, gradually deteriorating individuals' cognitive, physical, and social functions and requiring increasing degrees of care. There is a growing agreement that dementia treatment should initiate with nonpharmacological interventions to ameliorate challenging behaviors such as those aforementioned because (1) they address the psychosocial/environmental causes of 
the behavior and (2) they avoid the limitations of pharmacological interventions, namely, adverse side effects, drug-drug interactions, and limited efficacy [7]. Nonpharmacological interventions have been classified as (a) cognitive/emotionoriented interventions; (b) sensory stimulation interventions; (c) behavior management techniques; and (d) other psychosocial interventions such as occupational therapy (OT). Maximizing the effect of these interventions requires individualized intervention planning and execution according to the unique needs and strengths of the $\mathrm{PwD}$ [8]. There is evidence that OT is effective in dementia treatment $[5,7]$. The focus of OT is to improve $\mathrm{PwD}$ ability to perform activities of daily living promoting their independence, encouraging participation in social activities, and reduce the burden on the caregiver. Typical OT intervention involves the assessment of PwD abilities, training family caregivers in skills such as problem solving and coping strategies, and implementing environmental and compensatory strategies to assist the PwD in engaging in meaningful activities. Several studies have proven that multicomponent psychosocial interventions that are tailored and focused on the patient-caregiver dyad are the most effective in dementia [9]. Occupational therapists can play an important role in the care of the $\mathrm{PwD}$, given their expertise at understanding the complex relationships between person, environment, and occupation required for successful activity execution. Therapists accompany PwD during the course of the disease, providing education and skills training and supporting the caregivers.

Ambient intelligence (AmI) has been recognized as a promising approach for improving home and communitybased care aiming at mitigating dementia effects on individuals and families [10]. Ambient intelligence is a new paradigm in information technology aimed at empowering people's capabilities by the means of digital environments that are sensitive, adaptive, and responsive to human needs [11]. The development of AmI systems to support dementia treatment should consider that nonpharmacological interventions not only need to be adapted to the particular PwD and caregiver needs but also may need to evolve or change as the dementia progresses and that even new or more severe BPSD manifestations may appear. An ambient-assisted intervention system (AaIS) uses AmI to improve PwD's quality of life by identifying the presence of BPSD, deciding on an appropriate intervention and either modifying the environment or persuading the $\mathrm{PwD}$ or the caregiver to act on the system's advice [12]. We have implemented an assisted cognition system that follows the AaIS approach. This paper extends preliminary work incorporating an additional case of study for assessing the feasibility to support personalized occupational therapy interventions to address BPSD [13]. The results show evidence of the system's acceptance and effectiveness to ameliorate incidents of BPSD while reducing the caregivers' burden.

\section{Personalized Ambient-Assisted Occupational Therapy}

Assistive technologies (AT) have the potential to assist occupational therapist in gathering assessment data, executing interventions, and monitoring responses to therapy. However, studies have mainly centered in evaluating low-cost and common AT, such as the use of craft kits, medication dispensers with automatic reminders, and environmental aids and detectors [14]. There is limited evidence about the use and impact of computing-based AT to support occupational therapy. Most research is based on qualitative studies conducted to capture the perception of the stakeholders involved in the caring of $\mathrm{PwD}$, about the potential and limitations of computing-based AT. For instance, results from an interviewbased study conducted with occupational therapists emphasize the benefits of multimedia-based assistive technology to support temporal orientation for dementia sufferers, if and when it is adapted to their needs and abilities, which is relevant to the acceptance of a technology [15]. Similarly, from semistructured interviews conducted with family caregivers and occupational therapists, a set of needs and ideas for possible solutions were identified, such as virtual companions to promote social wellbeing and robots to enable $\mathrm{PwD}$ to complete tasks with which they have difficulties [16]. On the other side, results from surveys applied to art therapists who specialize in therapy for persons with dementia were used to inform the design of the Engaging Platform for Art Development (ePAD) to enable PwD independent access to art creation [17]. ePAD is customizable such that an art therapist can choose themes and tools that they feel reflect PwD needs and preferences. Usability results suggest that participants found $\mathrm{ePAD}$ engaging but did not perceive the prompts as being effective.

The AaIS approach consists in a set of autonomous and collaborative agents that implement the services depicted in Figure 1. Thus, a behavior analysis agent identifies BPSD episodes, which can be explicitly observed and reported by the caregiver, or, alternatively, they can be inferred by analyzing the information perceived from agents attached to sensors located in the environment or worn by the PwD. Agitation, for instance, is manifested via repetitive movement and verbal expressions such as shouting or continuous talk. As depicted in Figure 1, we have used the InCense platform [18], developed to facilitate the gathering of context data from the mobile phone's sensors, such as microphone, accelerometer, and GPS data, that can be used to infer an incident of BPSD. Finally, once there is evidence that the PwD is exhibiting a BPSD, a decision model agent selects a behavioral intervention, which will be enacted through the intervention enactment agent directing the ambient actuator agents to execute one of the following actions: (a) intervene directly to change the configuration of the physical environment; (b) communicate with the caregiver to recommend an action to perform; or (c) communicate with the $\mathrm{PwD}$ to suggest an activity or provide him with information that could change his current behavior. Our model for tailoring the AaIS services is supported by an ontology used to make the decision about the intervention to enact, as described in [12].

Following the AaIS approach, we implemented an assisted cognition system to support occupational therapy for PwD. The system's user interface is based on two components: AnswerBoard and AnswerPad. AnswerBoard is a public ambient display implemented on a touch screen 


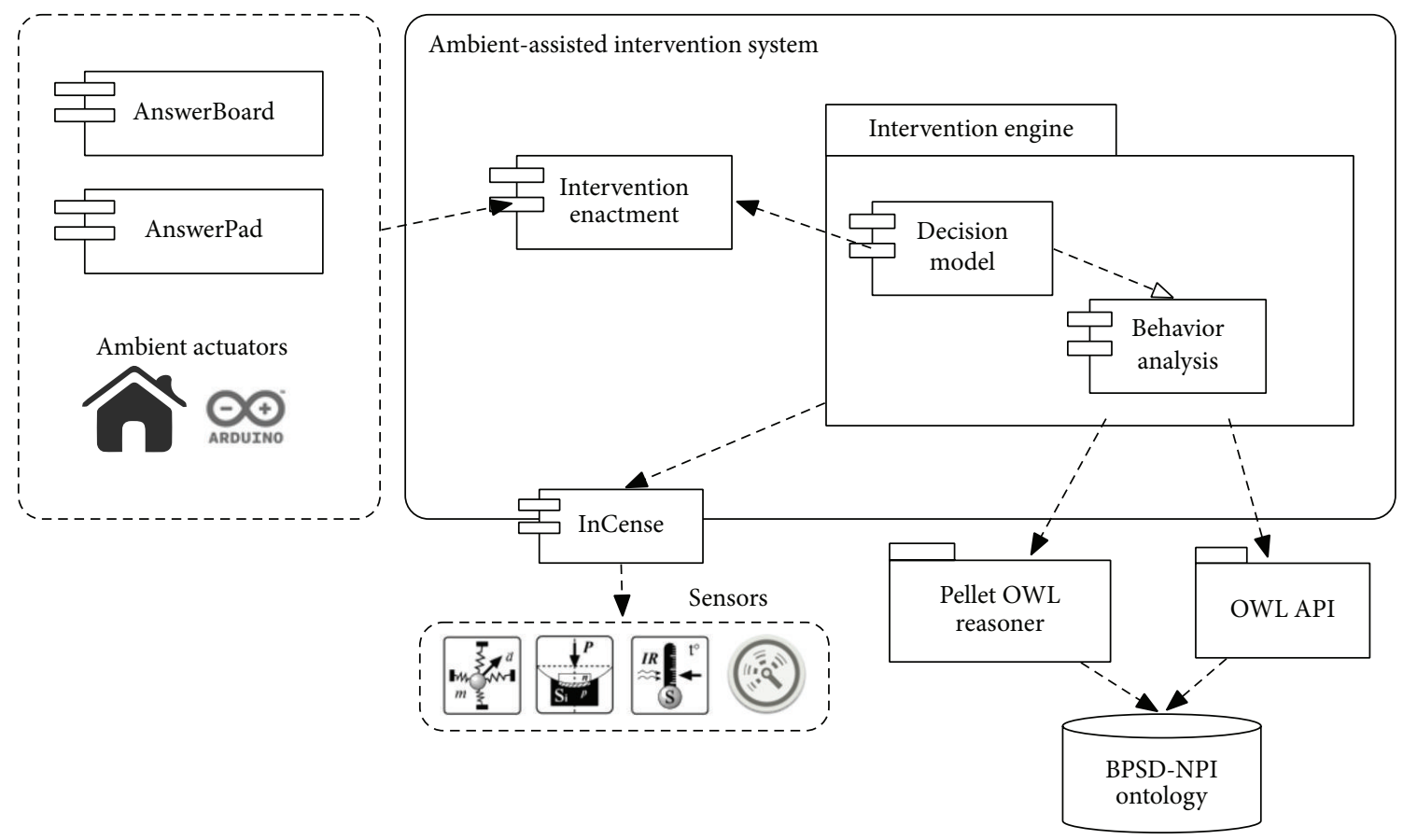

FIGURE 1: Ambient-assisted intervention system architecture.

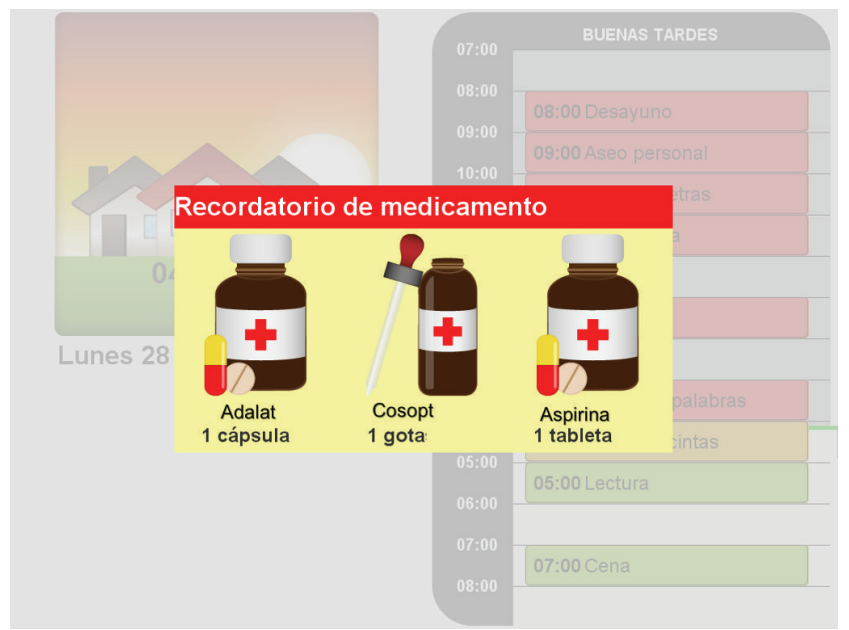

Figure 2: AnswerBoard showing a medication reminder.

LCD computer. Located in a common area within the PwD's home, it provides information of their activities for the current day, the current date, and time of day. Reminder messages are displayed on the AnswerBoard to prompt the patient on relevant events on his agenda, such as medication (Figure 2). The caregiver may create reminder notes from scratch or select one of the predefined templates completing the required information.

AnswerPad is an application running on an Android mobile phone with touch screen. It includes different widgets aiming to offer the PwD time and place awareness, reminder notes, and cues on his/her current activity and to maintain the connection with his/her social network. AnswerPad recollects data from the mobile phone's sensors to feed the intervention engine. Additionally, caregivers may use AnswerPad to manage elder's daily activities, keep track of his/her whereabouts, create reminder notes, and keep a diary of patient's behavior using an application.

\section{Evaluation}

This section describes a field study in which the effectiveness of the assisted cognition system AnswerPad/AnswerBoard to support occupational therapy interventions was evaluated with two case studies.

3.1. Study Design. The purpose of the study was to evaluate quantitatively and qualitatively to what extent the therapy system (a) promotes a positive response in the behavior of the PwD and (b) is adopted and found useful by the PwD and their caregivers. The inclusion criteria for PwD were elderly diagnosed with $\mathrm{AD}$, showing mild to moderate cognitive impairment (MMSE score between 14 and 24) and living in community. It was required that primary caregivers were family members living with the patient and not exhibiting physical or mental impairments that limit their role as caregivers. Two PwD and primary caregiver dyads were selected to participate in the study. In both cases, a common concern for the caregivers was the apathetic behavior of PwD. Apathy is a persistent disorder of motivation whose diagnostic criteria include (a) decreased motivation; (b) reduction of behavior, in particular, cognitive activity and goal-oriented motivation; (c) functional impairment attributable to apathy. Effective treatment of apathy, and other BPSD, requires a multifactorial approach, that is, a combination of different 
types of nonpharmacological interventions, as in the case of apathy, aimed at introducing new sources of satisfaction, interest, and encouragement. Also, it is recommended that interventions provide opportunities for socialization $[6,19]$.

The study involved home visits from a therapist to the participants' home during a period of 16 weeks. The therapist applied nonpharmacological interventions to address BPSD as suggested in clinical guidelines. Variables observed during the study were as follows:

(1) The presence and severity of challenging behaviors estimated by the scores of the Cumming's Neuropsychiatric Inventory Questionnaire (NPI-Q) to evaluate the effect of the intervention on the behavior in the PwD [20].

(2) The occurrence of apathy measured by the apathy evaluation scale (AES), an instrument developed to measure apathy resulting from neurological diseases [21].

(3) Caregiver burden, which is the psychological state resulting from the combination of physical, emotional, job, and social restrictions associated with caring for a sick person. In the study, the outcome of the Zarit Burden Interview (ZBI) was used to observe variations in the subjective burden reported by the caregiver [22].

(4) Caregiver self-efficacy, which refers to a subjective belief that a person has about his or her ability to successfully carry out certain kinds of caregiving tasks. This outcome was observed using the Revised Scale for Caregiving Self-Efficacy (RSCSE) [23].

Additionally, caregivers kept a diary of PwD behavior to document incidents they considered problematic, unusual changes in behavior, health status, and mood or memory problems. Caregivers reported each incident by describing the incident, the response of relatives to the incident, and the context in which it occurred (date and time). This information was reviewed on interviews with caregivers during follow-up visits, which enabled us to assess the effect of the intervention on the behavior of the participants. The study was divided into two stages:

(a) Intervention with Traditional Artifacts (Stage A). In this first stage, and for a period of 4 weeks, the therapist implemented a combination of strategies using traditional means, which included the use of external memory aids, cognitive training, reminiscence therapy, and techniques to enhance communication. Caregivers selected a set of activities that could be attractive for the person and easy to handle by them.

(b) Intervention Supported with AaIS (Stage B). In the second stage of the study, the AaIS supported the intervention. The AaIS services were tailored according to the particular needs of the participants. As in the first stage, the therapist conducted the sessions alternating the execution of activities supported by the therapy system with activities using traditional artifacts.
Visits were performed three times a week. The primary caregiver was involved in these sessions assisting the therapist. In each visit, the therapist guided the session consisting in the execution of three activities scheduled for the day. Each activity lasted a maximum time of 30 minutes to avoid fatigue on the participants. During the rest periods, 10 minutes between each activity, the therapist promoted communication between the PwD and their caregiver. The following sections describe the evaluation case studies.

3.2. Case Study S1. Daniel is an adult over 70 years of age diagnosed with Alzheimer's disease (MMSE =17). His primary caregivers are his wife Ana (66 years) and their daughter Sonia (43 years), who reside in the same household. They expressed particular concern about the lack of interest of Daniel for any type of activity during the day. They noted that most of the day Daniel is asleep in his room. Another concern for caregivers is the refusal of Daniel to take medication. They need to remind him of the medication schedule and supervise the intake, since repeatedly Daniel hid the pills in his pocket, or his mouth, for later disposal.

To address Daniel's apathy in a first stage of the intervention (Stage A), and in agreement with caregivers, the therapist defined a weekly schedule of activities that could be attractive for the PwD and easily handled by the caregivers. For instance, Daniel enjoyed solving crossword and Sudoku puzzles prior to the dementia onset. So, one of the activities involved solving crossword puzzles using pencil and paper. Another concern of the caregivers was of the PwD to take his medication. They need to remind him of the medication schedule and monitor the intake. Often Daniel hid the pills in his pocket or mouth for later disposal. Due to this situation, an external memory aids based intervention was implemented through a whiteboard $(40 \mathrm{~cm} \times 30 \mathrm{~cm})$ placed in the kitchen for displaying his medication schedule and the use of paper cards with written instructions for taking the medications contained in a labeled pill organizer.

In the second stage of the study (Stage B), the assisted cognition system supported the intervention. The AaIS services were tailored according to the particular needs of the participants. As in the first stage, the therapist conducted the sessions alternating the execution of activities supported by the system with activities using traditional artifacts. As part of the system deployment, AnswerBoard was installed on a 20-inch touchscreen computer over a table in the living room. AnswerBoard displays the agenda of activities previously defined in Stage A. To complete the deployment of the AaIS, two mobile phones running AnswerPad were given to Mr. Daniel and his caregivers. Using AnswerPad, the caregivers created and delivered medication reminders and other activities prompts, which Mr. Daniel would receive in his AnswerPad or in the AnswerBoard's screen. AnswerBoard included the implementation of two games: (a) Memorama and (b) Alphabet Soup.

(a) Memorama. It is a card game in which all the cards are laid face down on the touchscreen display and two cards are flipped face up over each turn (Figure 3(a)). The purpose of the game is to turn over pairs of matching cards. The user 


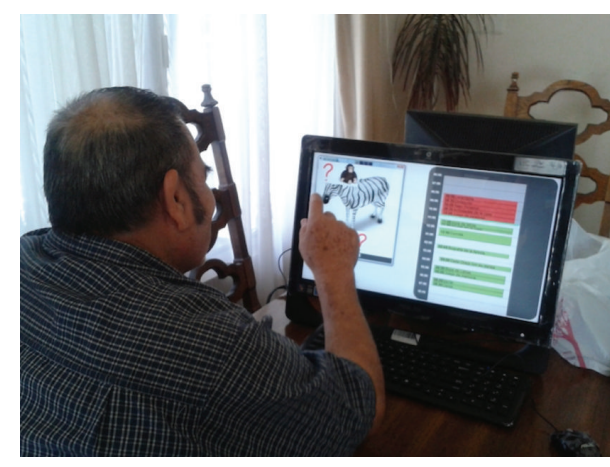

(a)

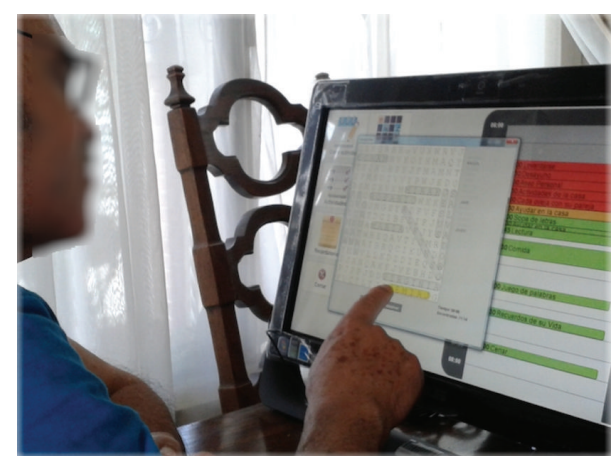

(b)

FIgURe 3: Mr. Daniel performing the activities implemented on AnswerBoard: (a) Memorama and (b) Alphabet Soup.

chooses two cards touching the screen to turn them face up. If cards show the same picture, the cards disappear displaying part of the background image. If they do not show the same picture, they are turned face down again. The game ends when the last pair of cards has been picked up.

(b) Alphabet Soup. In this activity, the participant is presented with a list of words that must be found in a grid of letters showing in the touchscreen display (Figure 3(b)). Each list has words of one of the following categories: (a) animals; (b) objects in the house; (c) months of the year; (d) names of family members; (e) fruit; and (f) countries. Each list has a maximum of 20 and a minimum of 10 words. Words have 7 letters on average and 10 as a maximum. Words can appear horizontally, vertically, or diagonally in the grid. Participants mark each found word dragging their finger over the word. If the word matches a word on the list, the word is highlighted and removed from the list.

Research has proposed matching activities to individual interests and retained skills to engage persons with dementia and maintain involvement [8]. The selection of games implemented was stirred by the adoption of similar activities that Mr. Daniel found enjoyable. During the first week, it was observed that Mr. Daniel experienced difficulties identifying and selecting the words in Alphabet Soup. The game interface was customized increasing the font size and the dimensions of the grid in which the letters are shown. Likewise, caregivers pointed out that a hear impairment in Mr. Daniel sometimes prevented him from hearing the reminders' audio notification in AnswerPad, so it was configured to vibrate whenever it received the reminder. In the following sections, we present the results obtained from the application of assessment instruments, interviews with caregiver, and system usage data obtained from the logs generated by the AaIS.

3.2.1. Results on Adoption and Usability. The average daily running time of AnswerBoard was 11:30 h. On average, the system started at 8:21 in the morning and was deactivated in the evening at around 19:56. The average number of days used in a week was 6.7 days. Figure 4 shows the average hours of daily use for each week of the Stage B of the study.

Figure 5 shows the number of reminders received by the participant through AnswerBoard classified into four

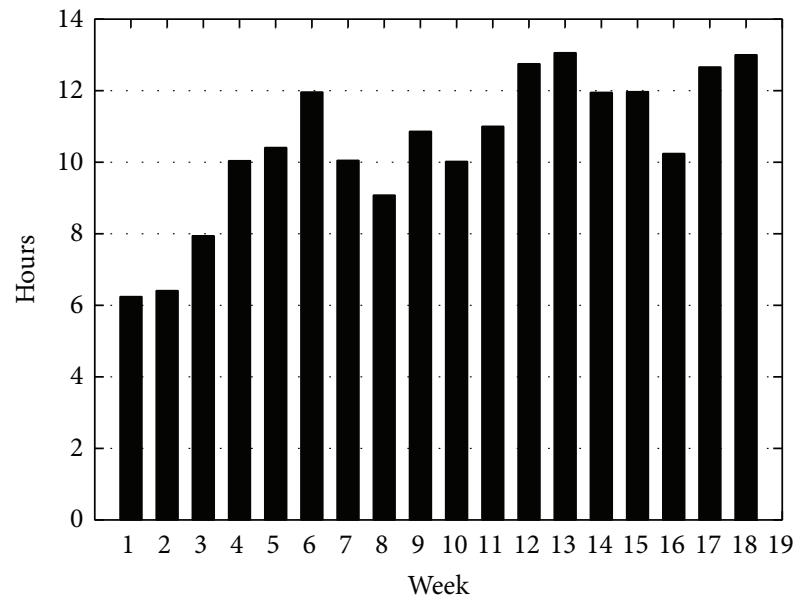

FIGURE 4: AnswerBoard average daily usage.

categories: (a) medications: reminders for medication; (b) activities: reminders about the activities on the agenda; (c) prompting: directions to support an activity; (d) orientation: reminder for temporal or spatial orientation. All the reminders were created and delivered through AnswerPad by the caregivers. As shown in Figure 5, the predominant type of reminder is for medication, except for the month of May, when the system was deployed only in the last week of the month. The decrease in the number of medication reminders in August is associated with the removal of medications prescribed to Mr. Daniel by his family physician.

With respect to the activities implemented through AnswerBoard, the daily use of Memorama averaged 29 minutes ( $\mathrm{SD}=42$ minutes), and on average the activity was performed 5 times a day $(\mathrm{SD}=4)$. The average time to complete a game was 6 minutes ( $\mathrm{SD}=16$ minutes). The Alphabet Soup activity average daily use was 37 minutes $(\mathrm{SD}=26$ minutes). The average daily usage was $6(\mathrm{SD}=4)$ times. On average, each activity required $37 \mathrm{~min}(\mathrm{SD}=42 \mathrm{~min})$ to finish.

\subsubsection{Results on Challenging Behaviors and Caregiver Burden.} This section presents the results obtained from the application of assessment instruments. Table 1 summarizes the results obtained from assessment instruments in 16 weeks of 


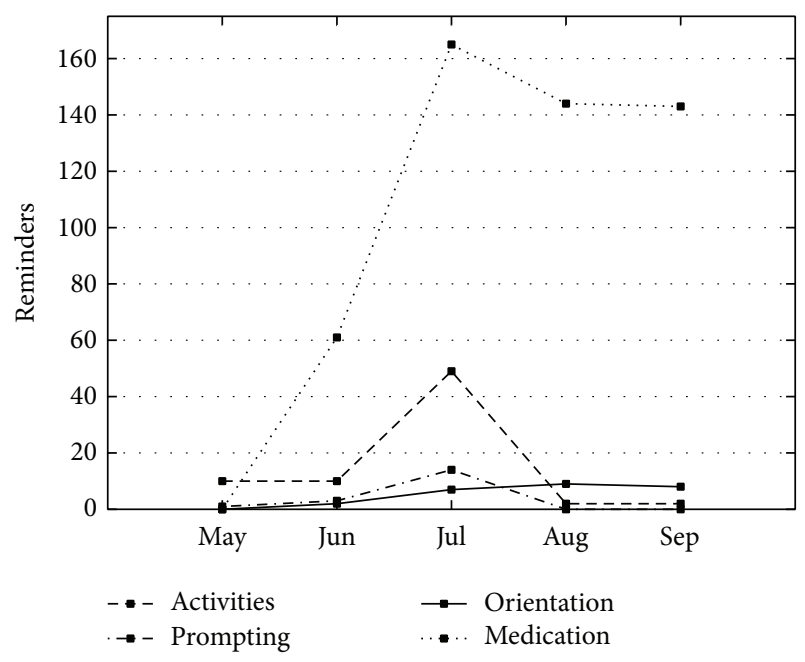

Figure 5: Reminders delivered to the PwD using AnswerPad.

TABLE 1: Assessment instruments result for case study S1.

\begin{tabular}{|c|c|c|c|c|c|c|c|c|}
\hline \multirow{2}{*}{ Instrument } & \multicolumn{2}{|c|}{ Apr } & \multicolumn{2}{|c|}{ May } & \multicolumn{2}{|c|}{ Jun } & \multicolumn{2}{|c|}{ Jul } \\
\hline & $\mathrm{C} 2$ & $\mathrm{C} 3$ & $\mathrm{C} 2$ & $\mathrm{C} 3$ & $\mathrm{C} 2$ & $\mathrm{C} 3$ & $\mathrm{C} 2$ & $\mathrm{C} 3$ \\
\hline Apathy AES & 64 & 54 & 63 & 54 & 60 & 50 & 49 & 51 \\
\hline NPI-Q total & 27 & 19 & 15 & 16 & 14 & 15 & 14 & 13 \\
\hline NPI-Q apathy subscale & 4 & 3 & 3 & 3 & 3 & 3 & 3 & 2 \\
\hline NPI-Q depression subscale & 0 & 4 & 0 & 3 & 2 & 3 & 3 & 2 \\
\hline Caregiver burden ZBI & 36 & 31 & 34 & 28 & 21 & 16 & 17 & 18 \\
\hline Caregiver self-efficacy RSCSE & 81 & 39 & 83 & 41 & 83 & 45 & 90 & 49 \\
\hline
\end{tabular}

the study. The results are shown in ordered pairs in which the first value corresponds to results reported by PwD's wife, Ms. Ana (C2), and the second to results reported by his daughter, Sonia (C3).

Throughout the study, Ana reported incidents in 7 of the 12 NPI-Q's subscales $(\mathrm{M}=17.5, \mathrm{SD}=5.5)$. Sonia reported incidents in 4 of the 12 NPI-Q's subscales $(\mathrm{M}=15.75, \mathrm{SD}=$ 2.17). The results of the apathy evaluation scale (AES) show a slight variation in the scores reported by both caregivers, Ana $(\mathrm{M}=58.20, \mathrm{SD}=5.56)$ and Sonia $(\mathrm{M}=53.20, \mathrm{SD}=2.48)$. The maximum score in the scale is 72 points. In $\mathrm{PwD}$, a score greater than 41.5 points indicates the presence of pathological apathy.

The Zarit Burden Interview (ZBI) has a maximum score of 88 points and questions are grouped into three categories: (a) consequences of care in the caregiver (11 questions, 0 44 points), (b) beliefs and expectations of their caregiving skills (7 questions, $0-28$ points), and (c) caregiver-patient relationship (4 questions, $0-16$ points). The average rating of the $\mathrm{ZBI}$ observed for Ana is 24 ( $\mathrm{SD}=9.44)$. The average rating of the ZBI observed for Sonia is $21.2(\mathrm{SD}=7.03)$.

The Revised Scale for Caregiving Self-Efficacy (RSCSE) measures the perceived ability of caregivers to deal with challenging behaviors of elders with cognitive impairment. The mean score reported by Ana is $84.13(\mathrm{SD}=3.05)$, which is considerably higher than the average scores reported by Sonia $(\mathrm{M}=47.47, \mathrm{SD}=8.98)$.
TABLE 2: Assessment instruments result for case study S2.

\begin{tabular}{lcccc}
\hline Instrument & May & Jun & Jul & Aug \\
\hline Apathy AES & 60 & 57 & 52 & 42 \\
NPI-Q total & 18 & 13 & 9 & 6 \\
NPI-Q apathy subscale & 9 & 6 & 2 & 2 \\
NPI-Q depression subscale & 3 & 2 & 2 & 2 \\
Caregiver burden ZBI & 38 & 36 & 30 & 32 \\
Caregiver self-efficacy RSCSE & 16 & 16 & 18 & 22 \\
\hline
\end{tabular}

3.3. Case Study S2. Julia (44 years) is the primary caregiver for Maria (73 years), her mother, who suffers from $\mathrm{AD}$ (MMSE $=21$ ). Maria also suffers from diabetes and hypertension, thus requiring the administration of different medications throughout the day. She often forgets what medication to take and at what time. Another concern for Julia is her mother's lack of motivation to perform her activities of daily living, such as eating, bathing, and dressing. She spends most part of the day sleeping, frequently getting out of the bed at midday. Maria often refuses to shower and Julia has to insist and eventually help her. Activities that Maria enjoys include caring for her dog and playing board games (i.e., cards, dominoes). Julia is also in charge of her father Raul (77 years) and husband of Maria. Raul is under treatment for cancer requiring attention but being able of performing activities of daily living independently. For this case, the intervention addressed the medication problems through automatic reminders and the apathy through occupational therapy.

For the first stage (Stage A) of the intervention, activities for home visits were defined in collaboration with her caregiver. Visits were held three days a week, which lasted on average 2 hours and were performed on the dining table. During the four weeks of Stage A, the activities were conducted with traditional artifacts (Figure 6(a)). In the second stage of the intervention (Stage B), the system was introduced for carrying out some activities, along with the traditional methods (Figure 6(b)). In both stages, the therapist kept a logbook to document the progress of the session.

3.3.1. Results on Adoption and Usability. With regard to the use of the assisted cognition system by participants, the average operating time was 13:03 h daily $(\mathrm{SD}=3: 14 \mathrm{~h})$. On average, caregiver activated the system in the morning at $4: 33 \mathrm{~h}(\mathrm{SD}=2: 52 \mathrm{~h})$ and it was turned off in the evening at around 19:06 $\mathrm{h}(\mathrm{SD}=2: 39 \mathrm{~h})$. On average, the system was used in 6.5 days a week.

3.3.2. Results on Challenging Behaviors and Caregiver Burden. Table 2 shows a summary of data collected with the assessment instruments during the 16 weeks of the study.

The assessment of problematic behaviors using the NPI$\mathrm{Q}$ shows an average score of 11.5 points $(\mathrm{SD}=4.5)$. Incidents were reported in 7 of the 12 subscales that make up the NPI. The three subscales with greater contribution to the total score are apathy $(\mathrm{M}=4.75, \mathrm{SD}=2.95)$, depression $(\mathrm{M}=$ $2.25, \mathrm{SD}=0.43)$, and changes in appetite/food $(\mathrm{M}=1.5$, 


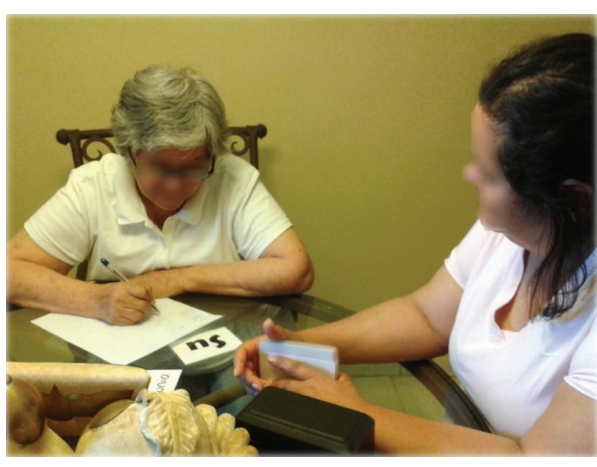

(a)

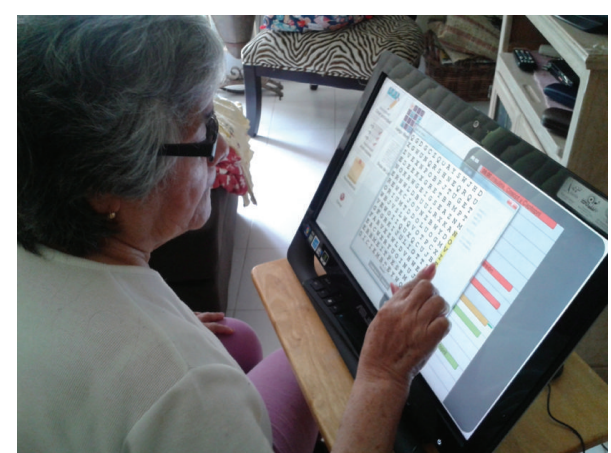

(b)

FIgURE 6: Ms. Maria performing activities (a) using traditional artifacts in Stage A and (b) using AnswerBoard in Stage B.

$\mathrm{SD}=0.87)$. The maximum score of the AES is 72 points; for people with dementia, a score over 41.5 points suggests the presence of pathological apathy. The average rating of the AES was 52.75 ( $S D=6.83)$. A Pearson product-moment correlation coefficient was computed to assess the relationship between AES and the subscale of apathy in the NPI scores. A high correlation $(r=0.84)$ is observed in the scores obtained by AES against the results of the subscale apathy NPI. In both scales, a reduction in the scores is observed throughout the study. The last measurement of the AES, 42, is close to the cutoff point for pathological apathy. Scores of the NPI apathy subscale suggest a reduction in both frequency and intensity of the incidents observed by the caregiver. The average score of the caregiver burden is $34(\mathrm{SD}=3.16)$. The correlation of the caregiver burden (ZBI) with the scores of apathy (AES) is high $(r=0.72)$. Also a very high correlation $(r=0.97)$ of the caregiver burden is observed with respect to the scores of the subscale apathy in the NPI.

Although the scores of the RSCE show a positive trend, the mean score is low $(\mathrm{M}=18, \mathrm{SD}=2.45)$ indicating that the caregiver has low confidence in his abilities as a caregiver. Caregiver burden and perceived self-efficacy have a high negative correlation $(r=-0.73)$. The correlation of apathy (AES) with respect to the perceived self-efficacy in care shows a very high negative correlation $(r=-0.99)$. The correlation of the apathy subscale of the NPI and the perceived selfefficacy in care is high $(r=-0.76)$.

\section{Discussion}

This section presents the analysis of the results described in the previous sections. The analysis focuses on the effects of the intervention on issues such as medication, apathy, overload/efficacy of caregivers, and adoption of the system. The discussion considers the quantitative and qualitative data obtained from interviews with caregivers, the logs kept by the therapist, and the logs generated by the system. Study results must be analyzed considering that the therapist home visits could have some effect on the behavior of the $\mathrm{PwD}$, affecting the dynamics between the caregiver and the $\mathrm{PwD}$, and therefore the results. However, this is a common trait among nonpharmacological interventions for dementia treatment [24].
Although older adults generally have a positive opinion about using technology [25], they are less likely to use the technology compared to younger individuals [26]. However, the leading factors that predict technology acceptance for older adults are usefulness and usability $[25,27,28]$. In both cases, the system was used consistently throughout the 16 weeks of observation. The usage logs generated by the system indicate that at least 12 hours a day the system was on. Also, the system log shows that the participants used the system almost everyday. These indicators of system adoption are strengthened by evidence gathered from interviews and session's logbooks home visits. The interface learnability is a key factor for usability. A system that is easy to use improves the user performance in task completion with the successive use of the system. One of the basic functions evaluated in the study was the intervention based on external memory aids implemented through touchscreen devices. From the analysis of data in the system log, it can be seen that participants reduced the response time to medication reminders and activity prompts. For instance, for case study S1, we observe that after the first four weeks of the intervention $29 \%$ of the reminders were cleared in less than 2 minutes, an action that requires the user to tap on the reminder on AnswerBoard and AnswerPad. After 16 weeks, $49 \%$ of the reminders were cleared in less than 2 minutes. Further evidence of the adoption of the system is derived from qualitative data gathered from interviews with the caregivers and the therapist's log: Ana (caregiver): I feel good because now I know he's taking his pills, not like before. He hid the pills and now he doesn't... I feel safer now because (he is?) already taking the pills. During home visits, the therapist observed that often when Mr. Daniel noticed the reminder, he headed to the kitchen for a glass of water, took the medication, and deactivated the reminder. Since reminders were shown on both devices, he commonly deactivated the AnswerBoard reminder first and later the reminder on AnswerPad, although sometimes he forgot to turn off the reminder on AnswerPad. For case study S2, we observe that after the first four weeks of the intervention $36 \%$ of the reminders were cleared in less than 2 minutes, and after using the system for 16 weeks $78 \%$ were cleared in less than 2 minutes. The home visits offered the opportunity to observe the response of Maria to medication reminders. The 
therapist observed that in all occasions Maria noticed the reminder when it was activated. Also, she answered correctly when asked about what medication she should take.

A challenge in the study of apathy is the dimensions involving the concept. Motivation and interest are latent variables, whose qualities and quantities must be inferred observing the patient's behavior [29]. For case study S1, as noted in the previous section, the quantitative variation in the results of apathy subscale of the NPI-Q shows a decrease during the study. Likewise, the assessment of apathy by AES shows a reduction in the scores reported by the caregivers. Although scores remain above the cutoff (41.5) suggesting the presence of apathy in $\mathrm{PwD}$, the results of the interviews provide evidence of increased motivation in Daniel: Ana: I usually see him more aware of all. More cheerful too. In a better mood. Because before he slept most of the day. He was very quiet; not talking, and now he asks questions; Sonia: He is more active now. He can last 2 to 3 hours playing. He is entertained and doesn't take naps. For instance, yesterday $I$ asked him to take a nap in the afternoon and he said: "No, I'll be here for a while". The decrease in the scores is more evident in case study S2. The subscale of apathy in the NPI indicates that before the intervention Maria showed severe apathetic manifestations most days of the week. The latest measurements indicate a reduction to at most one mild episode in a week. Also, the score of the last measurement with AES scale ( 42 points) is just above the cutoff point of the scale for pathological apathy.

According to the diagnostic criteria for apathy proposed in [29], the essential characteristic of apathy, diminished motivation, must be present for at least four weeks; second, two of the three dimensions of apathy (reduction of goal-directed behavior, goal-directed cognitive activity, and emotions) must also be present; and, thirdly, there must be identifiable functional impairments attributable to apathy. So the increase in motivation reported by caregivers suggests a positive effect of the intervention in reducing apathy. For instance, in case study S2, the caregiver comments (a) She [Maria] is more motivated, with better attitude, are more active. She does not object, doesn't grumble. Anything I ask her she shows willingness; (b) When visiting my brother she is interested in their children. Before, she did not ask for their grandchildren. Comments from caregiver in case study S1 include (a) Previously, he did not grab a broom or anything. Today in the morning he grabbed the broom and swept the garden. He moved the pots and swept. Now he does chores.

Caregivers of PwD require effective behavioral problem management strategies in order to keep them functional and reduce their own physical and emotional risks. Caregivers with high expectations of self-efficacy tend to continue to provide care even if the patient worsens their condition and care tasks become more demanding and perform their caregiver role with relatively lower burden [30]. The observed correlation between burden and self-efficacy in the study suggests such effect of the intervention on participants. During the study, caregiver burden levels show a decreasing trend, while levels of self-efficacy in caregivers increase. At baseline, the burden levels of caregivers in case study S1 were within the range of mild to moderate burden (20-40 points). After 8 weeks, burden level drops to mild burden (0-20 points). Throughout the study, the level of self-efficacy for both caregivers showed a positive trend, especially in the domain of response to problematic behaviors. In the case of Ana, an increase of $51.61 \%$ was registered in her confidence to respond adequately to problematic behaviors going from a baseline score of 62 to 94 after the 16 weeks of the study. Likewise, Ana's score increased $90.91 \%$ from the baseline score of 44 points to 84 points at the end of the intervention, suggesting a positive effect of the intervention in developing skills to deal with problematic behaviors by caregivers. Additionally, caregivers perceive a reduction in the dependence of Mr. Daniel. Some of the comments that support this are We used to be much more attentive to him. Not anymore, because now we can be in another place, doing chores, and he can be alone in the room. We felt calmer and more relaxed, because before, we always had to be at his side. The assessment of self-efficacy for the caregiver in case study S2 indicates a low level of confidence in her abilities to deal with the challenges posed by its role as primary caregiver. However, throughout the study, a positive trend can be seen in her perception of self-efficacy. After 16 weeks, there is an increase of $35.50 \%$ in RSCSE scale score with respect to the measurement at baseline. The self-efficacy domains that had a more favorable response were the ability to respond to problematic behavior (60\%) and the ability to obtain respite (57.14\%). With regard to the caregiver burden, even though the scores are within the range of mild to moderate burden, a slight reduction in the score along the study is observed.

\section{Conclusion}

In this paper, we report the results of an intervention with an assisted cognition system to support occupational therapy aiming at reducing PwD' challenging behaviors. Through an in situ study with two cases of PwD-caregivers dyads, we collected qualitative and quantitative evidence showing that our system was effective in helping the participants to be more independent and motivated to conduct daily activities. Responses from the caregivers indicated that they appreciated the potential of the system to make older adults more independent and responsible for taking medications, which helped to reduce caregivers' hassles. The impact of using the system was twofold: PwDs' incidents of apathy manifestations were reduced; and caregivers perceived that they improved their ability to conduct caregiving tasks. Overall experiences were consistently positive in both cases. It is recognized that PwD manifest the disease in very different ways $[2,5]$, making it difficult to extrapolate intervention outcomes from one case to the other. If anything, this emphasizes the need to personalize the intervention $[5,8,30]$, and an adaptable assisted cognition system can be of significant support in this.

Studies on the professional practice of occupational therapy have revealed that much more time is spent on assessment of the PwD (e.g., in some cases reaching 75\% of the time), rather than providing intervention [31]. This issue emphasizes the importance of developing more effective assessment tools. Future work involves developing a set of applications to support the occupational therapist in gathering sufficient 
and high-quality information from the $\mathrm{PwD}$ for intervention personalization.

\section{Competing Interests}

The authors declare that there is no conflict of interests regarding the publication of this paper.

\section{Acknowledgments}

This project was approved by the Institutional Ethics Committee with the approval ID\#2010-04. This work was partially supported by a grant from the Alzheimer's Association (ETAC-10-173237) and by CONACYT, Project 179795.

\section{References}

[1] A. Wimo and M. Prince, The Global Economic Impact of Dementia, Alzheimer's Disease International, London, UK, 2010.

[2] J. E. Galvin and C. H. Sadowsky, "Practical guidelines for the recognition and diagnosis of dementia," The Journal of the American Board of Family Medicine, vol. 25, no. 3, pp. 367-382, 2012.

[3] K. Rockwood, “Treatment expectations in Alzheimer's disease," The Canadian Review of Alzheimer's Disease and Other Dementias, vol. 9, pp. 4-8, 2007.

[4] S. Douglas, I. James, and C. Ballard, "Non-pharmacological interventions in dementia," Advances in Psychiatric Treatment, vol. 10, no. 3, pp. 171-179, 2004.

[5] A. K. Desai and F. Galliano Desai, "Management of behavioral and psychological symptoms of dementia," Current Geriatrics Reports, vol. 3, no. 4, pp. 259-272, 2014.

[6] S. Ishii, N. Weintraub, and J. R. Mervis, "Apathy: a common psychiatric syndrome in the elderly," Journal of the American Medical Directors Association, vol. 10, no. 6, pp. 381-393, 2009.

[7] C. H. Sadowsky and J. E. Galvin, "Guidelines for the management of cognitive and behavioral problems in dementia," The Journal of the American Board of Family Medicine, vol. 25, no. 3, pp. 350-366, 2012.

[8] L. N. Gitlin, N. Hodgson, E. Jutkowitz, and L. Pizzi, "The costeffectiveness of a nonpharmacologic intervention for individuals with dementia and family caregivers: the tailored activity program," American Journal of Geriatric Psychiatry, vol. 18, no. 6, pp. 510-519, 2010.

[9] N. Van’t Leven, M. J. L. Graff, M. Kaijen, B. J. M. de Swart, M. G. M. Olde Rikkert, and M. J. M. Vernooij-Dassen, "Barriers to and facilitators for the use of an evidence-based occupational therapy guideline for older people with dementia and their carers," International Journal of Geriatric Psychiatry, vol. 27, no. 7, pp. 742-748, 2012.

[10] P. Rashidi and A. Mihailidis, "A survey on ambient-assisted living tools for older adults," IEEE Journal of Biomedical and Health Informatics, vol. 17, no. 3, pp. 579-590, 2013.

[11] F. Sadri, "Ambient intelligence: a survey," ACM Computing Surveys, vol. 43, no. 4, article 36, pp. 1-66, 2011.

[12] R. F. Navarro, M. D. Rodríguez, and J. Favela, "Intervention tailoring in augmented cognition systems for elders with dementia," IEEE Journal of Biomedical and Health Informatics, vol. 18, no. 1, pp. 361-367, 2014.
[13] R. F. Navarro, M. D. Rodríguez, and J. Favela, "Use and adoption of a touch-based occupational therapy tool for people suffering from dementia," in Artificial Computation in Biology and Medicine, J. M. F. Vicente, J. R. Álvarez-Sánchez, F. de la Paz López, F. J. Toledo-Moreo, and H. Adeli, Eds., vol. 9107 of Lecture Notes in Computer Science, pp. 459-468, Springer, Cham, Switzerland, 2015.

[14] L. N. Gitlin, L. Winter, and M. P. Dennis, "Assistive devices caregivers use and find helpful to manage problem behaviors of dementia," Gerontechnology, vol. 9, no. 3, 2010.

[15] U. Ende and A. Sigmund, "An occupational therapeutic perspective about the usage of multimedia technologies in support of dementia," Gerontechnology, vol. 13, no. 2, p. 195, 2014.

[16] J. Boger, M. Quraishi, N. Turcotte, and L. Dunal, "The identification of assistive technologies being used to support the daily occupations of community-dwelling older adults with dementia: a cross-sectional pilot study," Disability and Rehabilitation: Assistive Technology, vol. 9, no. 1, pp. 17-30, 2014.

[17] V. Leuty, J. Boger, L. Young, J. Hoey, and A. Mihailidis, "Engaging older adults with dementia in creative occupations using artificially intelligent assistive technology," Assistive Technology, vol. 25, no. 2, pp. 72-79, 2013.

[18] M. Perez, L. Castro, and J. Favela, "InCense: a research kit to facilitate behavioral data gathering from populations of mobile phone users," in Proceedings of the 5th International Symposium of Ubiquitous Computing and Ambient Intelligence (UCAmI '11), pp. 25-34, Riviera Maya, Mexico, December 2011.

[19] H. Brodaty and K. Burns, "Nonpharmacological management of apathy in dementia: a systematic review," The American Journal of Geriatric Psychiatry, vol. 20, no. 7, pp. 549-564, 2012.

[20] J. L. Cummings, M. Mega, K. Gray, S. Rosenberg-Thompson, D. A. Carusi, and J. Gornbein, "The neuropsychiatric inventory: comprehensive assessment of psychopathology in dementia," Neurology, vol. 44, no. 12, pp. 2308-2314, 1994.

[21] D. E. Clarke, R. van Reekum, M. Simard, D. L. Streiner, M. Freedman, and D. Conn, "Apathy in dementia: an examination of the psychometric properties of the Apathy Evaluation Scale," The Journal of Neuropsychiatry and Clinical Neurosciences, vol. 19, no. 1, pp. 57-64, 2007.

[22] S. H. Zarit, P. A. Todd, and J. M. Zarit, "Subjective burden of husbands and wives as caregivers: a longitudinal study," The Gerontologist, vol. 26, no. 3, pp. 260-266, 1986.

[23] A. M. Steffen, C. McKibbin, A. M. Zeiss, D. GallagherThompson, and A. Bandura, "The revised scale for caregiving self-efficacy: reliability and validity studies," The Journals of Gerontology-Series B: Psychological Sciences and Social Sciences, vol. 57, no. 1, pp. P74-P86, 2002.

[24] J. Cohen-Mansfield, "Nonpharmacologic treatment of behavioral disorders in dementia," Current Treatment Options in Neurology, vol. 15, no. 6, pp. 765-785, 2013.

[25] T. L. Mitzner, J. B. Boron, C. B. Fausset et al., "Older adults talk technology: technology usage and attitudes," Computers in Human Behavior, vol. 26, no. 6, pp. 1710-1721, 2010.

[26] A. D. Fisk, W. A. Rogers, N. Charness, S. J. Czaja, and J. Sharit, Designing for Older Adults: Principles and Creative Human Factors Approaches, CRC Press, Boca Raton, Fla, USA, 2009.

[27] D. G. Bouwhuis, "Design for person-environment interaction in older age: a gerontechnological perspective," Gerontechnology, vol. 2, no. 3, pp. 232-246, 2003.

[28] N. Selwyn, "The information aged: a qualitative study of older adults' use of information and communications technology," Journal of Aging Studies, vol. 18, no. 4, pp. 369-384, 2004. 
[29] P. Robert, C. U. Onyike, A. F. G. Leentjens et al., "Proposed diagnostic criteria for apathy in Alzheimer's disease and other neuropsychiatric disorders," European Psychiatry, vol. 24, no. 2, pp. 98-104, 2009.

[30] C. S. Mackenzie and G. Peragine, "Measuring and enhancing self-efficacy among professional caregivers of individuals with dementia," American Journal of Alzheimer's Disease and Other Dementias, vol. 18, no. 5, pp. 291-299, 2003.

[31] J. Tung, H. Snyder, J. Hoey, A. Mihailidis, M. Carrillo, and J. Favela, "Everyday patient-care technologies for Alzheimer's disease," IEEE Pervasive Computing, vol. 12, no. 4, pp. 80-83, 2013. 


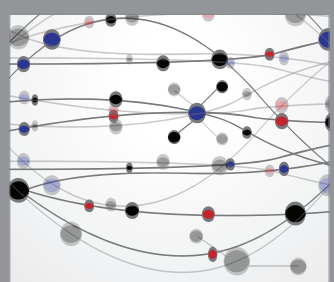

The Scientific World Journal
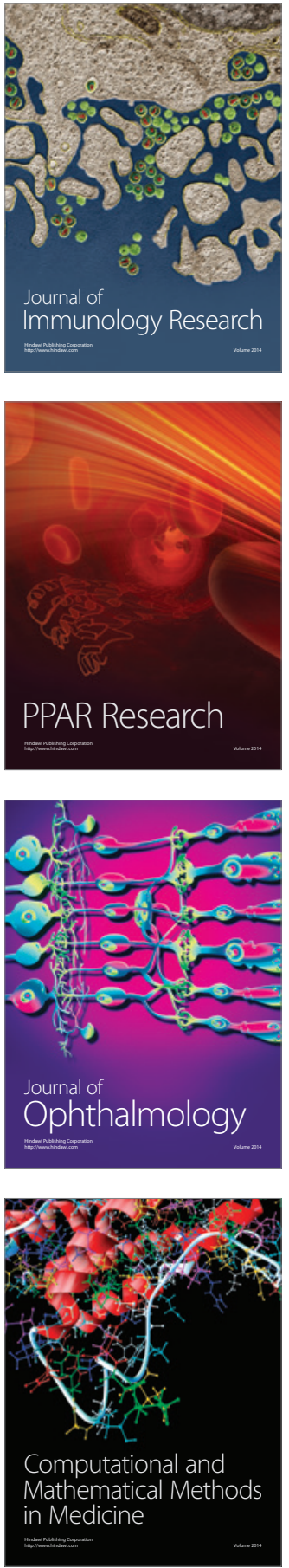

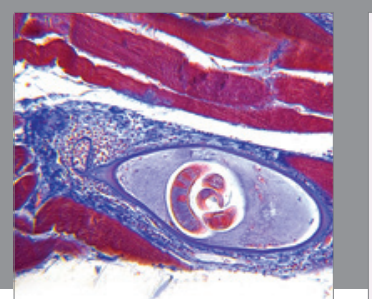

Gastroenterology Research and Practice

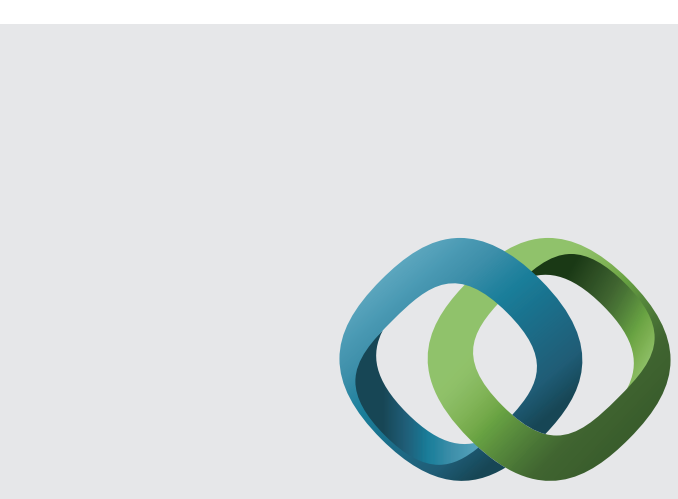

\section{Hindawi}

Submit your manuscripts at

http://www.hindawi.com
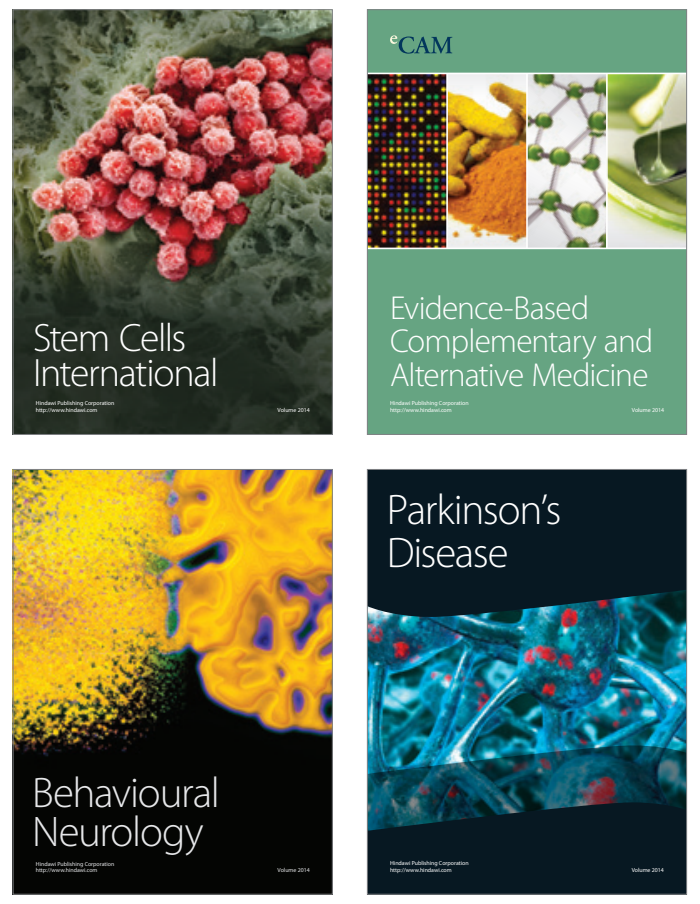
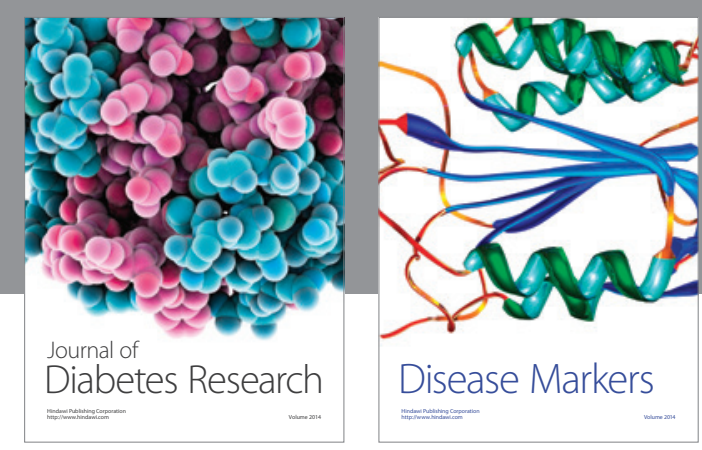

Disease Markers
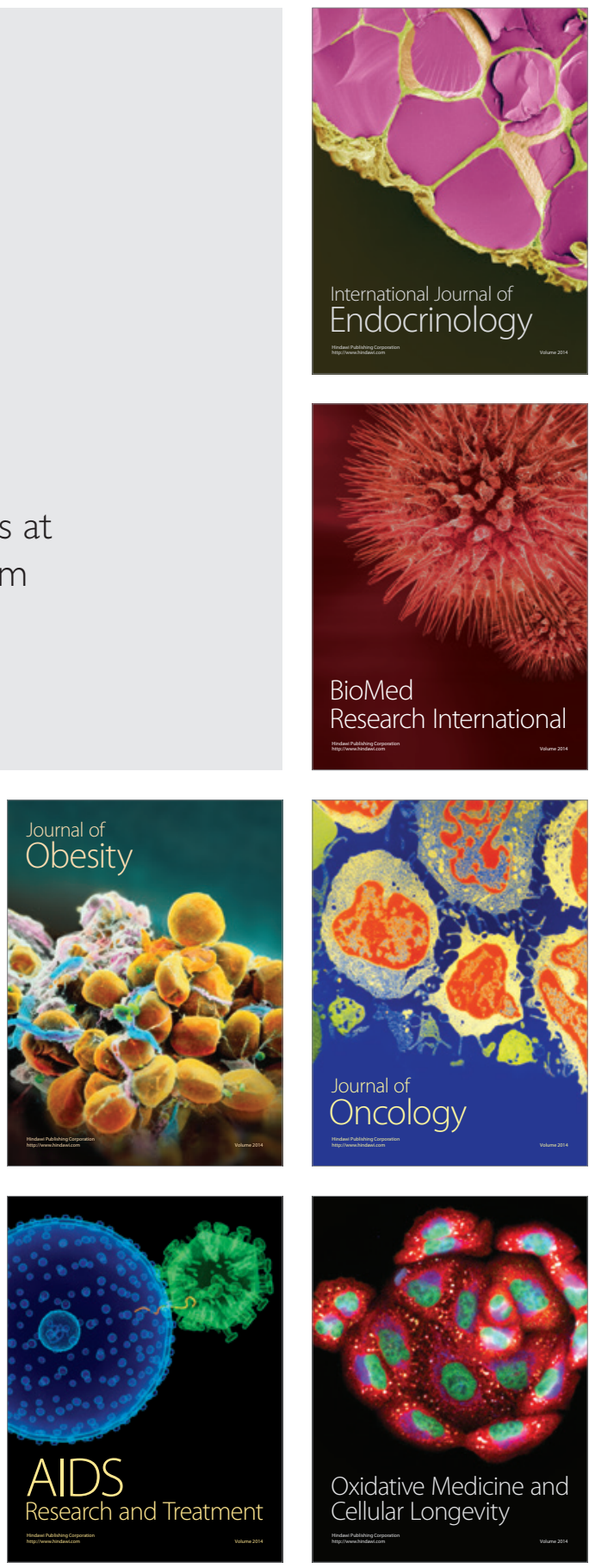